\title{
Un projet pour les écoles isolées
}

Rui Canário

\section{(2) OpenEdition}

\section{Journals}

Édition électronique

URL : http://journals.openedition.org/ries/3330

DOI : $10.4000 /$ ries.3330

ISSN : 2261-4265

\section{Éditeur}

Centre international d'études pédagogiques

\section{Édition imprimée}

Date de publication : 1 juin 1996

Pagination : 111-120

ISSN : 1254-4590

\section{Référence électronique}

Rui Canário, "Un projet pour les écoles isolées », Revue internationale d'éducation de Sèvres [En ligne],

10 | 1996, mis en ligne le 30 juillet 2013, consulté le 14 novembre 2019. URL : http:// journals.openedition.org/ries/3330 ; DOI : 10.4000/ries.3330

Ce document a été généré automatiquement le 14 novembre 2019.

(c) Tous droits réservés 


\title{
Un projet pour les écoles isolées
}

\author{
Rui Canário
}

1 Depuis 1991, se développe au Portugal un projet d'intervention éducative en milieu rural qui s'adresse aux petites écoles primaires situées dans des régions marquées par l'isolement, le déclin démographique, le vieillissement des populations, la perte d'une identité séculaire. Le « Projet des écoles isolées » essaye de construire, du point de vue de l'école, avec les acteurs locaux (instituteurs, enfants, familles, élus locaux, etc.), des réponses éducatives plus pertinentes, par rapport au contexte singulier de chaque communauté. Il s'agit d'un projet national, conduit sous l'égide de l'Institut des communautés éducatives (ICE), financé par la fondation Bernard Van Leer (Hollande), soutenu au point de vue logistique par le ministère de l'Éducation nationale.

\section{Quel futur pour les petites écoles rurales?}

2 La naissance et le développement, en Europe, des systèmes scolaires publics sont contemporains de l'émergence et de la construction des États modernes, libéraux, qui sont venus se substituer à l'ancien régime. La mise en place d'un très dense réseau d'écoles primaires sur tout le territoire national, notamment dans les régions rurales, a joué un rôle très important dans un processus d'unification culturelle et politique.

3 L'école primaire a constitué, par rapport au monde rural, un véhicule de pénétration d'une culture urbaine et laïque. Pourtant, si, dans un premier moment, la réaction a été surtout défensive, dans un second moment, les communautés villageoises se sont appropriées l'école primaire. Finalement, l'école primaire a acquis une dimension emblématique et elle est devenue un symbole de progrès et un élément identitaire de chaque village. Comme le disent Guindani et Bassand (1982) : " une commune sans école n'est plus une commune ».

4 Au Portugal, comme ailleurs en Europe, l'avenir des petites écoles en milieu rural est très fortement menacé. Le débat tend à s'organiser autour de deux questions essentielles : d'une part, on pose le problème des coûts économiques des écoles de très petite dimension, d'autre part, on questionne la "qualité » pédagogique du service rendu par 
ces écoles. C'est au nom de cette double rationalité (économique et pédagogique) qu'une politique de fermeture des écoles trouve ses fondements.

Pourtant, si, pour quelques-uns, la petite école en milieu rural est condamnée à disparaître, au nom de la " modernité » et du « développement », d'autres ont cherché à mettre en évidence le fait que la fermeture des écoles, en général accompagnée de la fermeture d'autres services publics, peut avoir l'effet d'accélérer, et même de rendre irréversible le déclin des communautés villageoises.

6 Débattre de l'avenir des petites écoles en milieu rural, signifie questionner l'avenir et la survivance du monde rural, dans son ensemble. Dans cette perspective, s'impose un réexamen critique des modèles de société et de développement, encore dominants à la fin du XXe siècle. Le «Projet des écoles isolées" (envisagé comme un projet d'intervention éducative et sociale) correspond à un pari qui se fonde sur une conviction double : d'un côté, on admet que l'école en milieu rural est susceptible de devenir un pôle de revitalisation des zones rurales, d'un autre côté, on pense qu'elle pourra, simultanément, contribuer à une rénovation des pratiques pédagogiques et éducatives.

\section{Le réseau : une alternative à la fermeture}

7 Conçu comme une alternative à une politique de fermeture systématique des écoles et de concentration des enfants loin de leurs petits villages, le "Projet des écoles isolées", selon Rui d'Espiney (1994), visait dès son début deux finalités principales : tout d'abord, contrarier la situation d'isolement dans laquelle se trouvaient les enseignants, contrainte majeure de leur action pédagogique, lourde de conséquences aux plans personnel et professionnel; d'autre part, on voulait contribuer à éviter le processus de progressive "périphérisation » de ces écoles et, donc, des communautés locales dans lesquelles elles s'inscrivent.

8 Le problème des " petites écoles » en milieu rural, qui n'est pas spécifique du Portugal (Canário, 1995), est très loin de correspondre à une question mineure : au Portugal, plus de la moitié des écoles primaires ont un ou deux postes d'enseignant, dont un pourcentage significatif a moins de dix élèves. Cette situation, malgré la politique de fermeture, est restée stable pendant les dix dernières années. En effet, la baisse constante du taux de natalité fait toujours « naître » de nouvelles écoles de petites dimensions.

9 Le «Projet des écoles isolées » est présent sur l'ensemble du territoire national (cinq régions) et sa dimension peut être appréciée à partir des données relatives à l'année 1995: il y a un total de cent quatre-vingt-quinze écoles concernées par le projet, correspondant à cent quatre-vingts petits villages. Les écoles sont organisées par petits groupes (quarante groupes constitués d'après un critère de proximité géographique). Le total d'instituteurs et d'élèves correspond, respectivement, à deux cent soixante-huit et trois mille six cent cinquante. Sur le terrain, sont partenaires actifs du projet cinq établissements de l'enseignement supérieur, vingt-sept mairies et cent quatorze Juntas (petites communes qui constituent l'unité administrative la plus petite, chaque mairie comporte plusieurs Juntas).

10 Chaque petit groupe d'écoles (trois à six) s'organise comme un réseau, dont les activités communes et les échanges s'alimentent des projets d'action autonomes que chaque établissement développe, en cherchant à établir des liens pertinents et fertiles entre les activités scolaires et les dynamiques de l'action communautaire. Malgré son point de 
départ, le projet ne se laisse pas enfermer dans des frontières strictement scolaires. Au contraire, sa vocation - et son critère de réussite - est, finalement, celui de s'intégrer dans un projet éducatif plus vaste qui puisse se confondre avec un processus de développement local. d'intervenants extérieurs qui est appelée à jouer un rôle de formation/animation. La diversité des initiatives est la règle, d'ailleurs très fortement encouragée et valorisée. Malgré cela, il y a des activités communes à l'ensemble des écoles concernées qui ont joué un rôle très important dans la construction de l'identité du projet. C'est notamment le cas des «jours différents » : ils sont organisés, tout au long de l'année scolaire, selon une périodicité variable, par chaque groupe d'écoles.

Il s'agit d'organiser une journée de travail (et aussi de loisir), qui se déroule dans une école qui fonctionne comme " hôtesse ", à laquelle participent tous les instituteurs et tous les élèves de l'ensemble du réseau. La réalisation de cette journée de travail suppose la coopération de partenaires extérieurs aux écoles, en particulier les familles et les élus locaux.

13 Le "jour différent " constitue une opportunité de rompre l'isolement, de mettre en relation des enfants de différents villages, d'organiser des activités pédagogiques basées sur les échanges et la valorisation des ressources locales, de créer une situation formative pour les enseignants (préparer et/ou faire le bilan du «jour différent», construire et échanger des matériaux pédagogiques, étudier comment réinvestir les activités du « jour différent» dans chaque salle de classe). Chaque école, au-delà des «jours différents ", maintient des contacts réguliers avec les écoles voisines (et des écoles d'autres groupes) en utilisant d'autres voies, comme par exemple la correspondance scolaire. Au niveau national, l'ICE, dans sa fonction de pilotage, assure la circulation de l'information, organise des réunions de travail entre les équipes d'intervenants extérieurs, assure la réalisation annuelle d'un colloque national avec la participation des enseignants, élus locaux, éléments des communautés locales, chercheurs en éducation.

\section{L'école et la communauté locale}

Du point de vue d'un État « éducateur » et centraliste (Chariot, 1994), la mise en place d'un réseau dense d'écoles publiques a été envisagée comme une condition pour unifier et homogénéiser, en diffusant un enseignement identique sur tout le territoire national. Cette homogénéisation, parfois entendue (de façon erronnée) comme une stratégie pour garantir l'égalité des chances, implique que les spécificités locales puissent être ignorées. C'est dans cette perspective qu'on peut soutenir l'idée (Nóvoa, 1992) que l'institution scolaire est née d'un processus de rupture avec les communautés locales.

Le «Projet des écoles isolées » a comme point de départ l'idée selon laquelle une école fermée sur elle-même n'est en mesure de résoudre aucun problème important. Le rapport entre l'école et son environnement immédiat (physique, social, culturel, politique) devient un point critique essentiel qu'il faut être capable de remettre en équation. Il ne s'agit pas, surtout, d'« ouvrir " l'école à son milieu environnant à partir de stratégies didactiques, qui restent prisonnières du paradigme scolaire (Vincent, 1994). Il s'agit plutôt d'avoir la capacité de développer des démarches collaboratives de type partenarial 
avec les autres partenaires sociaux et éducatifs qui œuvrent dans une même unité territoriale et sociale (Barbier, 1995 ; B. Canário, 1995 ; Zay, 1995).

D'autre part, la construction d'une relation interactive, permanente, entre l'école et son environnement correspond, elle-même, à une stratégie de changement de l'institution scolaire. Pour rendre plus claire la nature des activités développées par les écoles, dans le cadre de ce projet, nous allons recourir à quelques exemples empruntés à l'expérience d'une région (le nord de l'Alentejo), en utilisant un texte dont l'auteur est Abilio Amiguinho (1995).

Premier exemple: Deux écoles, de deux villages voisins, ont réussi à concrétiser la reconstitution d'un ancien marché public que, comme ailleurs, le «progrès » et la «modernité » avaient fait disparaitre. Il s'agit de récupérer la mémoire d'une pratique sociale qui, auparavant, matérialisait les rapports de solidarité et de voisinage entre les habitants des deux communes.

Deuxième exemple: La fermeture des services publics contribue fortement à accélérer l'exode rural et la mort des communautés. À partir de la question « Où est le facteur?", trois petites écoles ont mené un projet commun pour recréer un service de distribution postale. L'action convergente des acteurs locaux (élèves, instituteurs, élus locaux, population) a réussi à faire revenir le facteur, particulièrement nécessaire pour éviter aux personnes âgées des déplacements constants. L'école a regagné une nouvelle signification aux yeux des élèves et de la population locale.

19 Troisième exemple: La valorisation des personnes âgées (envisagées comme une importante ressource et non plus comme un fardeau) constitue un des axes de travail du projet, dans cette région. Dans un petit village, l'école a pris l'initiative, avec la coopération de la Junta, d'une association locale et, bien sûr, avec l'implication directe des intéressés, de transformer une salle de classe (maintenant vide) en centre d'accueil pour le troisième âge.

Quatrième exemple : L'école d'un petit village a décidé de défier la bureaucratie des services culturels de l'État et elle a pris l'initiative, avec l'appui et la coopération d'autres partenaires, de mettre sur pied un musée local, en contribuant de cette manière à l'enrichissement de l'environnement culturel. Le musée a été installé dans un édifice récupéré qui avait fonctionné, auparavant, comme une prison.

Cinquième exemple : Dans un autre petit village, le curé s'est associé au projet de travail de l'école, dont la visée était d'étudier et, si possible, récupérer les traditions locales. Auparavant, la principale occupation des habitants du village consistait à exploiter les mines. En articulation avec l'école, le curé a réussi à organiser une procession en l'honneur de la patronne des mineurs. Cette fête religieuse n'avait pas eu lieu depuis cinquante ans.

Tous ces exemples nous permettent de mettre en évidence un certain nombre de traits communs. Tout d'abord, ils révèlent que l'institution scolaire, dont le rôle «reproducteur» a été fortement souligné par les sociologues de l'éducation, participe, simultanément, d'un processus de production du social. Elle peut donc être aussi une source d'innovations sociales et éducatives. C'est justement dans la mesure où l'école réussit dans son activité à "transgresser " les frontières traditionnelles du "scolaire " qu'elle pourra mieux contribuer à valoriser les savoirs et les pratiques culturelles locales. La fonction d'animation locale devient, à ce titre, une fonction structurante, susceptible de faciliter l'émergence de ce qu'Alberto de Melo désigne comme «une culture du 
développement » (1991). Dans cette perspective, l'école peut apparaître comme un pôle de développement et de revitalisation du tissu social en milieu rural.

Un autre aspect important consiste dans la globalisation de l'action éducative. En gardant la nécessaire spécificité du "travail scolaire», les écoles s'orientent vers son enrichissement, à partir d'un élargissement de son espace d'intervention, de la diversité des partenaires de l'action éducative, d'un changement qualitatif des pratiques pédagogiques en direction des enfants, envisagés comme la communauté locale à l'intérieur de l'école. Si l'éducation des enfants n'est pas réduite à l'action didactique exercée par les instituteurs, elle s'affirme, plutôt, comme le résultat d'une interdépendance de l'action et des apports de différentes institutions et de différents partenaires.

Cette vision « écologique " (Furter, 1981) invite à une convergence et à une articulation de modalités éducatives formelles et informelles, à une articulation entre les activités scolaires et extra-scolaires, à une articulation entre l'éducation des enfants et celle des adultes. De cette synergie entre différentes modalités, institutions et acteurs éducatifs, qui est tributaire de l'importance stratégique accordée à l'informel (Pain, 1990), résulte le renforcement du potentiel éducatif de l'environnement local, c'est-à-dire de son « éducogénie ». En cherchant à faire la synthèse des dimensions sociales de l'éducation et des dimensions éducatives du changement social, on arrive à faire coïncider un projet éducatif globalisé, avec un projet globalisé de développement local. De ce point de vue, l'enseignant devient aussi un agent du développement local.

Un troisième trait commun, important, correspond à la manière d'envisager le processus de rénovation pédagogique. Dans le cadre du «Projet des écoles isolées», le point de repère fondamental de toute l'intervention éducative est constitué par le triangle qui unit l'école, les familles, l'environnement local. Le fonctionnement de ce triangle apparaitt comme la clé pour la rénovation des pratiques pédagogiques qui deviennent plus pertinentes par rapport à leur environnement social et culturel. L'école peut regagner une nouvelle légitimité sociale, basée sur un «sens" nouveau du travail scolaire. La multiplication des situations éducatives qui permettent aux enfants d'apprendre devient la fonction essentielle de l'enseignant qui apparaît, alors, comme un producteur de sens. On évolue, de cette façon, de ce qu'on a appelé « un enseignement actif » vers ce qu'on peut appeler un « apprentissage interactif ».

De façon parallèle, à un enseignant qui devient un producteur de sens devra correspondre un élève producteur de savoirs. C'est de cette façon que, dans le cadre du «Projet des écoles isolées ", nous envisageons le changement de l'école à partir d'une interaction avec la communauté locale. L'enfant (plus qu'un « élève ») s'affirme comme un être curieux qui apprend à lire et à changer le monde, à partir d'une démarche investigative, en interaction avec ses pairs et avec les différentes sortes d'adultes.

Il n'est plus envisagé comme un «client » de l'école, encore moins comme une « matière première » à transformer. Il devient un travailleur qui a son " métier » (Perrenoud, 1994). Il ne s'agit pas de rénover l'école, en reniant ce qui est universel dans les savoirs et les compétences à acquérir par la voie scolaire, mais de combiner la voie de l'expérimentation avec la voie de la formalisation. Cette perspective est la seule permettant de considérer les personnes qui se forment (dans ce cas, les enfants) comme la principale ressource de leurs apprentissages. 

partenariat (Zay, 1995). Ce qui est réellement en cause, c'est la possibilité de construire des nouvelles modalités d'action, dans lesquelles l'école s'engage avec une pluralité de partenaires locaux. Cet engagement est concomitant avec le processus de changement de l'institution scolaire (B. Canario, 1995). La démarche partenariale, nécessairement fondée sur une convergence d'intérêts, s'inscrit dans une perspective d'investissement du local et de diffusion de la fonction formation, ce qui permet à la fois «une variété des partenaires et la cohérence d'ensemble de leur intervention » (Barbier, 1995).

La démarche partenariale, susceptible d'assumer plusieurs formes différentes, suppose toujours, pourtant, la mise en place d'un ou plusieurs réseaux, concept que Merini propose d'être défini de la façon suivante : «le réseau est la structure de liaison utilisée pour agencer les champs d'intervention et d'expertise des partenaires et mettre ainsi en synergie un certain nombre d'actions et de décisions» $(1995$, p. 7). Or, la démarche partenariale et la création de réseaux, à multiples niveaux (local, régional, national), sont au cœur même du « Projet des écoles isolées ».

L'action convergente de plusieurs partenaires individuels (enseignants, enfants, parents, élus locaux, chercheurs) et institutionnels (école, mairie, Junta, université, entreprise, association locale, centre de santé) devient une condition nécessaire pour qu'on puisse donner un sens nouveau au travail scolaire, à partir d'une pertinence accrue des activités éducatives qu'on propose aux enfants.

\section{Réinventer l'école}

31 Dans le débat, toujours vif, sur l'avenir des écoles en milieu rural, les défenseurs d'une solution qui passe par leur progressive et inéluctable fermeture avancent, en règle générale, deux arguments principaux : le premier concerne les coûts et, donc, le besoin d'arriver à la possiblité de faire des économies d'échelle ; le deuxième consiste à remettre en cause la possibilité, pour un milieu (la petite école rurale) aussi "pauvre », d'être capable de rendre un service éducatif public de "qualité ». Une étude empirique publiée récemment, conduite par Alain Mingat et Cédric Ogier (1995) vient contrarier ces deux arguments.

D'une part, l'étude montre qu'une politique de fermeture systématique des petites écoles n'est pas nécessairement plus avantageuse, d'un point de vue strictement économique. D'autre part, l'étude vient mettre en évidence les effets pédagogiques très positifs du fonctionnement des classes uniques, marquées par l'hétérogénéité. Il s'agit justement d'une caractéristique des petites écoles en milieu rural. Sans en retirer des conclusions définitives ou dogmatiques, les auteurs soulignent l'existence d'un large éventail de choix possibles pour orienter les politiques éducatives en milieu rural, notamment en ce qui concerne le réseau scolaire.

Les contraintes qui pèsent sur le quotidien des petites écoles sont, le plus souvent, évoquées pour démontrer l'impossibilité de leur survivance. L'expérience, au Portugal et ailleurs, montre que les contraintes peuvent devenir des ressources. C'est-à-dire que la proximité, la taille (réduite) et l'hétérogénéité des classes peuvent devenir des points d'appui pour produire des pratiques pédagogiques nouvelles (Bouchat, 1995; Collot, 1994). 

encourage, contribuent à transformer la petite école en milieu rural, selon le terme de Calvi (1995), en une sorte de « laboratoire » d'une école nouvelle. Dans cette perspective, la question de la « qualité » correspond, très souvent, à une fausse question, parce qu'on fait l'omission des rapports « écologiques » entre l'école et son environnement, en dehors desquels il n'est pas pertinent, ni possible, de faire a priori une définition de la " qualité " (Furter, 1981). enseignants et de son articulation avec la production délibérée de changements éducatifs, remplaçant celle qui a été dominante depuis les années soixante. Les vagues successives de réformes, conduites de façon autoritaire et verticale, ont consisté, pour l'essentiel, dans un processus d'exportation de solutions du "centre " (l'administration) vers la "périphérie» (les écoles). Les établissements ont été envisagés comme un terrain d'application, tandis qu'on assignait à la formation un rôle instrumental orienté vers la compétence individuelle des enseignants.

Dans le "Projet des écoles isolées ", nous essayons d'établir une rupture avec cette vision de rationalité technique du changement aussi bien que de la formation: on envisage les enseignants comme des professionnels qui se forment d'après un processus collectif d'intelligence des situations de travail (Barbier, 1992). D'un autre point de vue, les écoles deviennent les lieux principaux où les enseignants apprennent (Ingvarson, 1990). Donc, les enseignants et les écoles doivent changer de façon concomitante. L'adoption d'une stratégie de formation centrée sur l'école prend alors toute sa pertinence.

Les intervenants extérieurs cherchent justement à créer des dispositifs de formation qui puissent faire coïncider les espaces et les temps de travail avec les espaces et les temps de formation. La conception, l'exécution et le bilan d'un " jour différent» constituent, par exemple, une situation de travail qui se confond avec une situation formative. La même chose se passe quand un expert se déplace dans une salle de classe pour appuyer de façon clinique l'enseignant. Cette voie rend possible une interaction féconde entre l'action (résoudre des problèmes), l'innovation (produire des nouvelles) et la formation (changer la vision du monde).

\section{La solution et le problème}

La fermeture des petites écoles en milieu rural est présentée comme une solution. Mais quel problème viendrait-elle résoudre?

La fermeture des petites écoles rurales, envisagée comme une solution universelle, correspond à une démarche de "rationalisation " du réseau scolaire qui est tributaire d'une vision très réductionniste de la situation: il s'agit de réaliser une intervention interne au système scolaire qui vise à donner une réponse à une question technique, en ignorant (ou sous-estimant) ses dimensions sociales et culturelles. D'autre part, l'effort pour délimiter et circonscrire le problème à une dimension technique, en fait, apporte des complications, puisqu'il n'y a jamais de problèmes isolés susceptibles d'être résolus, un par un, selon une suite linéaire.

40

La situation des petites écoles en milieu rural est marquée par la complexité et, donc, elle fait appel à une approche globale. Nous ne sommes pas face à un problème scolaire qui découle d'une décroissance du nombre d'élèves, mais plutôt face à une situation 
problématique (Schon, 1983) qui n'est pas dissociable du caractère périphérique des zones rurales, de leurs pertes démographiques, des bas taux de scolarisation, de l'absence d'emploi, de la perte de l'identité culturelle.

41 Le mérite principal du « Projet des écoles isolées » a consisté dans sa capacité de proposer une autre façon de voir, et donc de construire le problème : nous ne sommes pas face à un problème de réseau, ou d'isolement des instituteurs, ou même d'isolement des écoles. Il s'agit, fondamentalement, d'un problème d'isolement des communautés villageoises. C'est pourquoi, comme nous le rappelle Yves Jean (1995), une rénovation de l'école en milieu rural peut devenir une condition pour l'émergence d'une nouvelle ruralité.

\section{BIBLIOGRAPHIE}

Amiguinho Abílio, Um testemunho em torno da problemática e da intervenção na escola rural (texte inédit), 1995.

Barbier J.-M., « La recherche de nouvelles formes de formation par et dans les situations de travail », Éducation permanente, 112 p. 125-145.

Barbier J.-M., « Tendances d'évolution de la formation et place du partenariat », in : Estado actual da investigação em formação, Porto, SPCE, 1995.

Bouchat T.-M., « Um dia tipo numa pequena escola rural », in: Canario Rui (Ed.), A escola rural na Europa, Setûbal, ICE, 1995.

Calvi, J.-M., « A escola, uma estrutura em osmose corn o meio ambiente », in: Canário Rui (Ed.), A escola rural na Europa, Setúbal, ICE, 1995.

Canário Rui (Ed.), A escola rural na Europa, Setúbal, ICE, 1995.

Canário M. - Beatriz, « Partenariado local e mudança educativa », Inovação (8) 1 et 2, p. 151-166.

Charlot Bernard (Ed.), L'école et le territoire : nouveaux espaces, nouveaux enjeux, Paris, A. Colin, 1994.

Collot Bernard, « Escola rural: uma opção », in: D’Espiney Rui (Ed.), Escolas isoladas em movimento, Setúbal, ICE, 1994.

D’Espiney Rui, (Ed.), « Uma problemàtica de rede escolar ou pedagogica? », in: D’espiney Rui (Ed.), Escolas isoladas em movimento, Setúbal, ICE, 1994.

Furter Pierre, « Diversités régionales ou réduction des inégalités dans le développement récent de l'éducation ", in : Carron G. et Ta Gnoc Châu (Eds), Disparités régionales dans le développement de l'éducation. Un problème controversé, Paris, Unesco, 1981.

Guindani Silvio, Bassand Michel, Maldéveloppement régional et identité. Pour un développement endogène, Lausanne, Presses polytechniques romandes, 1982.

Ingvarson Lawrence, « Schools: Places where teachers learn », in: Chapman Judith (Ed.), Schoolbased Decision-making and Management, London, Falmer Press, 1990.

Jean Yves, « Estado, escola e crise dos espaços rural », in: Canário Rui (Ed.), A escola rural na Europa , Setúbal, ICE, 1995. 
Melo Alberto, « Educação e formação para o desenvolvimento rural », Forum, 9/10, p. 149-160.

Mérini Corine, Le partenariat en formation : espace d'interculturalité, Communication au colloque « Formation, savoirs professionnels et situations de travail », Aipelf/Afirse (section portugaise), Lisbonne, université de Lisbonne (pol.), 1995.

Mingat A., Ogier C, « Elementos para uma reflexão nova sobre a escola primària em melo rural », in: Canário Rui (Ed.), A escola rural na Europa, Setübal, ICE 1995.

Nóvoa Antonio, « Para uma anlise das Instituicões escolares », in: Nóvoa Antonio (Ed.), As organizações escolares em análise, Lisboa, D. Quixote/ IIE, 1992.

Pain Abraham, Éducation informelle. Les effets formateurs au quotidien, Paris, L'Harmattan, 1990.

Perrenoud Philippe, Métier d'élève et sens du travail scolaire, Paris, ESF, 1994.

Schon Donald, The Reflective Practitioner. How Professionals Think in Action, London, Basic Books, 1983.

Vincent Guy (Ed.), L'éducation prisonnière de la forme scolaire? Scolarisation et socialisation dans les sociétés industrielles, Lyon, Presses universitaires de Lyon, 1994.

Zay Danielle, «L'école partenaire. Concept et dispositif », in : A escola, um objecto de estudo, Actas do $V$ colóquio da secção portuguesa da Aipelf/Afirse, Lisboa, Afirse, 1995.

\section{RÉSUMÉS}

Le projet d'intervention éducative et sociale mené au Portugal assigne à l'école en milieu rural un double rôle déterminant: dans le développement local, comme pôle de revitalisation sociale et de partenariat, et dans le système éducatif, comme lieu de rénovation pédagogique.

\section{INDEX}

Index géographique : Portugal

Mots-clés : développement rural, école rurale, milieu rural, rénovation de l'école

\section{AUTEUR}

\section{RUI CANÁRIO}

Professeur d'université, Instituto das comunidades educativas, Setubal, Portugal. 